\title{
A.C. LOSS CONTRIBUTIONS OF THE TRANSPORT CURRENT AND TRANSVERSE FIELD CAUSED BY COMBINED ACTION IN A
} MULTIFILAMENTARY WIRE

\author{
J.L. de Reuver, G.B.J. Mulder, P.C. Rem, and L.J.M. van de Klundert. \\ Twente University of Technology, P.O.B. 217, \\ 7500 AE Enschede, The Netherlands
}

\section{Abstract}

A.c. losses caused by an a.c. transport current and a transverse a.c. magnetic field during simultaneous action were measured. The loss contributions have been obtained separately. The measurements were performed on a NoTi multifilamentary wire having a CuNi matrix of low conductivity in order to prevent eddy currents. The test configuration is presented and measurement results as well as theoretical confirmation are dealt with.

\section{Introduction}

The combined exposure to a.c. transport current and a.c. field has been previously studied $[1,2]$, However, in these papers, the a.c. transport current is assumed to be either distributed uniformly over the filaments or in some cases taken to be fully localised in the outer filaments. The research dealt with in this paper emphasises the actual a.c. transport current and the corresponding a.c. transport current and magnetization losses. For the purpose of measuring the losses, a test configuration was developed with the option that the a. c. field amplitude can be varied independently. The design objective was to obtain an a.c. field in a relatively small volume in order to reduce the self-inductance. Coupling between a.c. coil and d.c. field was satisfactorily avoided. Several compensation techniques have been applied in order to obtain an optimal resolution.

\section{Theory}

The constitutive equation in the case of the combined action of an a.c. transport current and transverse a.c. magnetic field for a multifilamentary wire can be given by the expressions:

$$
\begin{array}{ll}
\bar{j}=n j_{C} \frac{\overline{\mathrm{E}}_{z}}{|\dot{\mathrm{B}}| \mathrm{R}_{\mathrm{f}}{ }^{*}} & \left|\overline{\mathrm{E}}_{z}\right| \leq|\dot{\mathrm{B}}|_{\mathrm{f}}{ }^{*} \\
\bar{j}=n j_{C} \operatorname{sgn}\left(\overline{\mathrm{E}}_{z}\right) & \left|\overline{\mathrm{E}}_{z}\right| \geq|\dot{\mathrm{B}}|_{\mathrm{R}} \text { * }
\end{array}
$$

with $R_{f}{ }^{*}=(\pi / 4) R_{f}, R_{f}$ is the radius of the filament and $B$ the external a.c. Field at the location of the filament. Expression (1) is only valid if full penetration of the applied field occurs. $\bar{j}$ is the mean current density over a filament and its surrounding matrix and $\bar{E}_{z}$ is the mean electric field.

The partial differential equation for the unsaturated case (1) is expressed by:

$$
\frac{1}{r} \frac{\partial}{\partial r}\left(r \frac{\partial}{\partial r} j\right)=\frac{\mu_{0} \eta j}{R_{f}{ }^{*}|\dot{B}|} \frac{\partial j}{\partial t}
$$

If we consider $B(t)=K I(t)$, the analytical result for the a.c. transport current losses per period per unit of length is given by:

$Q=\frac{-2 \mu_{0}}{R} \sqrt{ } \frac{K \tilde{I}_{f}{ }^{*}}{\pi \mu_{0} \eta j_{C}} \sum_{k=0}^{\infty} \frac{8 \hat{I}^{2}}{\pi^{4}(2 k+1) 7 / 2} F\left(R / \frac{(2 k+1) \pi \mu_{0} \eta j_{c}}{2 k R_{f}{ }^{*}}\right)$

$F \equiv \frac{\operatorname{Ber}_{0} B e r_{1}-\text { Ber }_{0} B e i_{1}+B e r_{1} B e i_{0}+B e i_{0} B e i_{1}}{B e r_{1}^{2}+B e i_{1}^{2}}$

and $R$ the radius of the wire.

This expression can be extended for dependence of the critical current density on the magnetic induction [3].

In the case of the fully localised current distribution the equation given by London [4] is applicable with $j=n j c$. If jc depends on $B$, numerical calculations can be performed to obtain the losses in this fully saturated case [5].

Regarding the intermediate cases, if both expressions (1) and (2) hold for different positions inside the wire, numerical calculations yield the losses [3].

It is shown that it is possible to describe the normalized loss quantity $\pi Q / \mu_{0} \hat{\mathrm{I}}^{2}$ by two parameters only, namely $i=\hat{I} / I c$ and $b=\pi R_{f}{ }^{*} \hat{B} /\left(\mu_{0} I c\right)$. The results of both analytically and numerically calculated losses are shown in figure 1. The limit of the validity of the

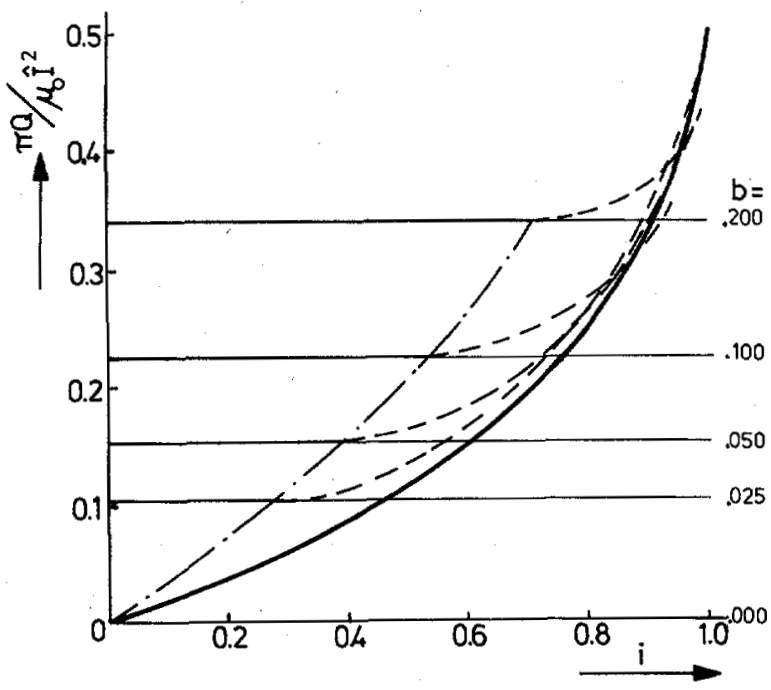

Figure 1. Normalized theoretical a.c. transport current losses in applied a.c. fields versus the current for various field amplitudes. The limitation of expression (4) is given by the centre line -..-. The numerical results are given by dashed lines ---. 
analytical expression (4) is indicated. For i values largex than this limit saturation appears for a certain b value.

The magnetization losses were calculated using the following approximation of the local magnetization as function of the external field.

$$
M=\frac{4}{3 \pi} \eta j_{c} R_{f}\left(1-\left(\frac{j}{\eta j}\right)_{c}^{2}\right)^{3 / 2}
$$

From this expression the total magnetization losses per cycle and unit of length can be obtained by integration over both the volume and applied field.

\section{Test arrangement}

\section{The a.c. magnetic field}

The field is obtained by a set of two coaxial solenoids with opposite current directions. The test field is generated in the gap between the solenoids. The selfinductance of this system is low compared with that of a single solenoid of the same size. The test configuration is given in figure 2 . In order to prevent coupling be-

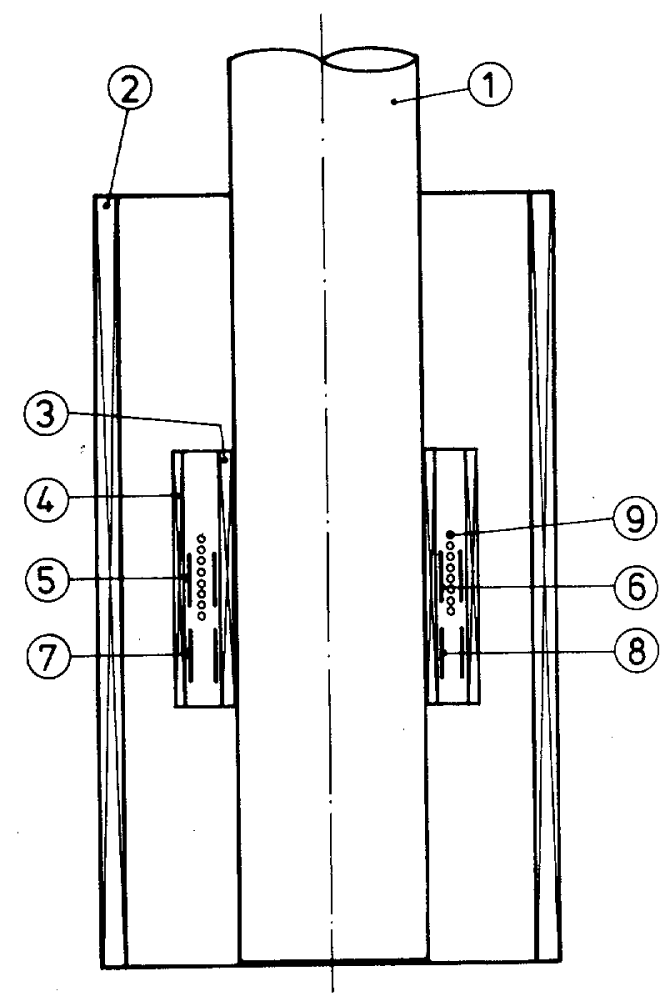

Figure 2, The measuring apparatus.

1) Bobbin

2) D.C. coil

3) Inner a.c. coil

4) Outer a.c. coil
5) Outer pick-up coil

6) Inner pick-up coil

7) Outer compensation coil

8) Inner compensation coil tween the d.c. coil and the a.c. field the inner a.c. coil has 3050 windings in 10 layers and the outer coil 2440 windings in 8 layers. The gap between the coils is $3 \mathrm{~mm}$ and the height of the coil pair $50 \mathrm{~mm}$. The test field is homogeneous within $2 \%$ for $96 \%$ of the available test volume. The disturbance of the a.c field caused by the coupling with the d.c. coil is $2 \%$.

The coaxial pick-up coils for measuring the magnetization losses, have a gap of $2 \mathrm{~mm}$ and surround part of the bifilary-wound test wire?. The spacing of the windings of the test wire is $1 \mathrm{~mm}$ in order to prevent electro magnetic interaction between the test windings. These coaxially-wound pick-up coils and the in-series compensation coils are made of wire having a conductivity of $2.10^{6}(\Omega \mathrm{m})^{-1}$ a radius of $50 \mu \mathrm{m}$ and a length of $12 \mathrm{~m}$ so that the eddy current losses in this pick-up coil wire are smaller than $0.1 \%$ of the losses in the test wire. Full compensation is obtained by means of a toroidal transformer in series with the a.c. coil, providing the time derivative of the a.c. field. Avoltage caused by a minor coupling between pick-up coil and the field of the a.c. transport current circuit in order to obtain a better resolution.

The a.c. transport current

The a.c. transport current is induced in a closed loop of the test wire by means of a transformer. The voltage across the test wire is measured by voltage terminals [5]. A compensation loop in series with the terminal wire is situated outside the a.c. coil. Full compensation with respect to the transport current is achieved by means of the Rogowski pick-up coil. In this case compensation with respect to the a.c. field is also desired because the area between the test wire and the terminal wire is intersected by the a.c. field. The toroidal transformer response is used for this purpose. A minor intersection of the area between the test wire and the terminal wire by the demagnetization field was also observed. Subtraction of the pick-up voltage from the loss voltage is necessary if small currents and large field amplitudes are used in order to achieve sufficient accuracy.

Measurement set-up

Trapezsidal ramp currents and fields were applied. Compensation is facilitated by assuming a continuous voltage response at the start of a ramp. Althoughthis approach may not be quite satisfactory with respect to the voltage response, minor variations in the compensation do not affect the loss values.

A computer generates the ramp, records the relevant responses and performs the integration required to obtain the loss values. The ramp times for $-\hat{\mathrm{B}}$ to $+\hat{\mathrm{B}}$ and $-\hat{\mathrm{I}}$ to $+\hat{\mathrm{I}}$ and the waiting period are all $22 \mathrm{~ms}$. The sample time is $400 \mu \mathrm{s}$.

Data of the test wire

The test wire is a MCA NbTi multifilamentary composite wire consisting of 114 filaments embedded in a matrix of CuNi. The diameters of the wire and the filaments are $200 \mu \mathrm{m}$ and $12.7 \mathrm{\mu m}$ respectively. $R_{f}^{*}$ is therefore $5 \mathrm{\mu m}$ and $n=0.45$. The effective critical current density $n j c$ has been measured and found to be $4.110^{9} \mathrm{Am}^{-2}$ and $2.110^{9} \mathrm{Am}^{-2}$. The corresponding cri. tical currents are $128 \mathrm{~A}$ and $65 \mathrm{~A}$ and the fields of first full penetration $47 \mathrm{mT}$ and $23 \mathrm{mT}$ respectively. 


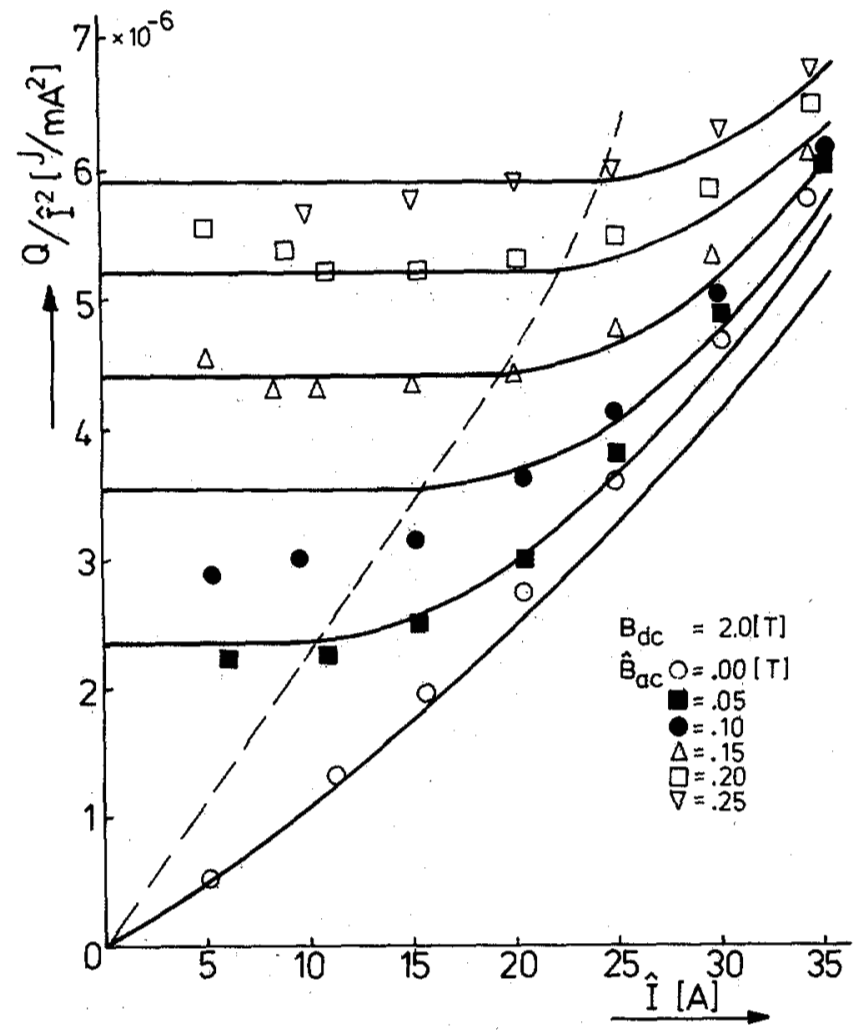

Figure 3. Measured and calculated a.c. transport current losses versus the a.c. transport current amplitude for various applied a.c. Field amplitudes with a background field for $2 T$.

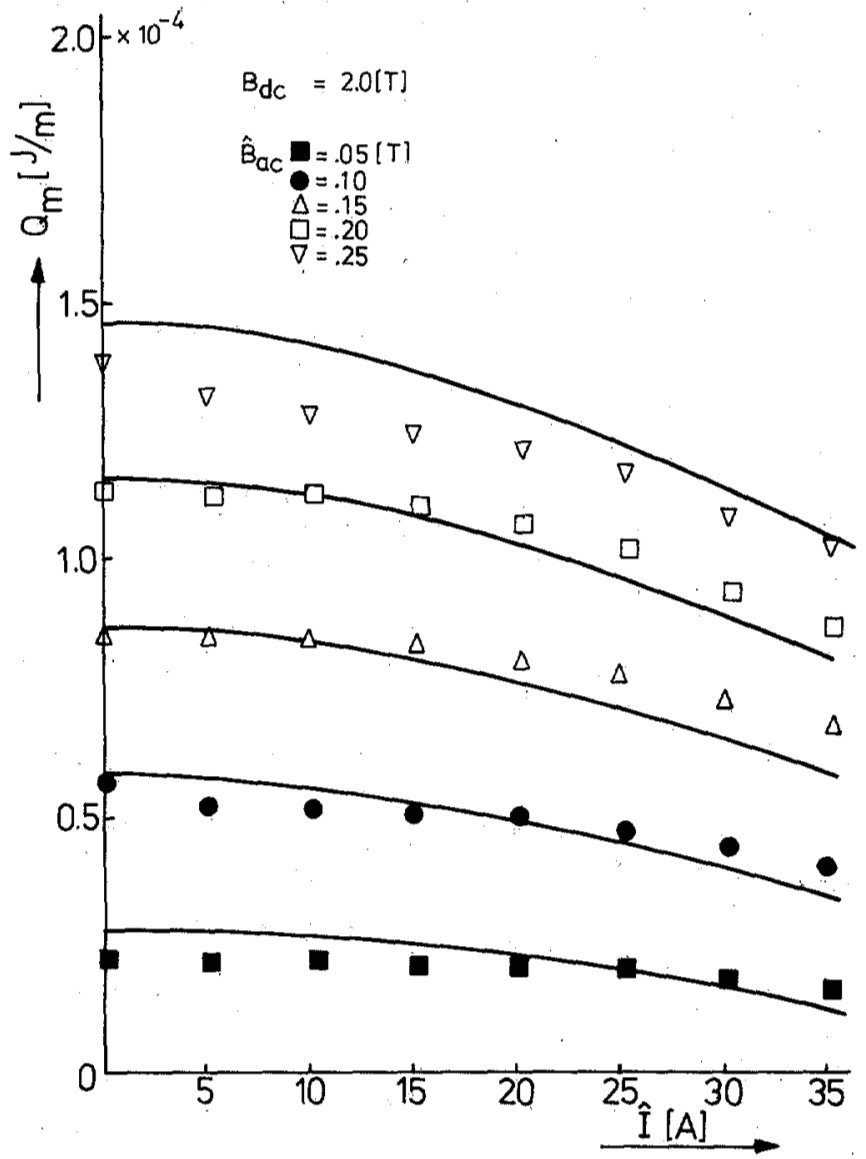

Figure 4. Measured and calculated magnetization losses versus the a.c. transport current amplitude for various applied a.c. field amplitudes with a background field of $2 \mathrm{~T}$.

\section{Results and discussion}

The a.c. transport current losses are shown in figures 3 and 6 for the a.c. fields of 0.8 and $2.0 \mathrm{~T}$. The jc(B) dependence is taken into account for both cases. In the case of $2 \mathrm{~T}$ no significant differences can be observed. The agreement between theory and experiment is satisfactory which proves the validity of loss results given in figure 1 and in particular shows the behavioux of the current density in the unsaturated region to be quasi resistive. From this figure we can derive useful information about the transition of the fully unsaturated case into the partially saturated one. This is helpful for the purpose of suppressing self-field instabilities.

The magnetization losses are shown in figures 4 and 7. A reduction of the magnetization losses can be observed with increasing a.c. transport current amplitide.

The combined losses are shown in figures 5 and 8 . In all cases the losses increase with increasing a, $c$. transport current or a.c. field amplitude. In the case of $2 \mathrm{~T}$ the contribution of the a,c. transport current losses is $30 \%$ of the magnetization losses for $\bar{I}=0 \mathrm{~A}$. However, this is already achieved for $i \simeq 0.5$; a considerable contribution which will be larger for types of wire with smaller filaments and the same $j c$.

\section{Conclusions}

It is shown that it is possible to accurately determine the a.c. transport current losses and the transverse a.c. magnetic field losses separately by the described measurement method.

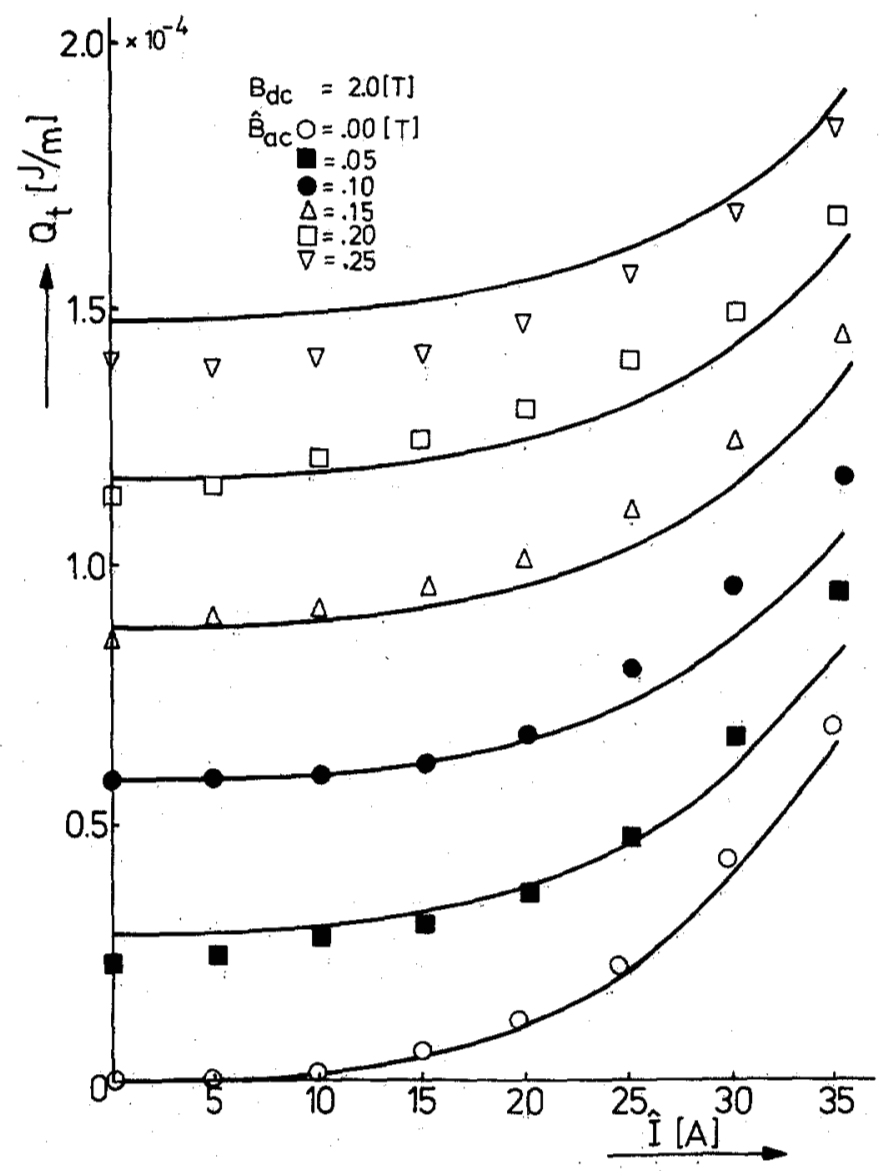

Figure 5. Measured and calculated combined losses of both a.c. transport current and a.c. field versus the a.c. transport current amplitude for various applied a.c. field amplitudes with a background field of $2 T$. 


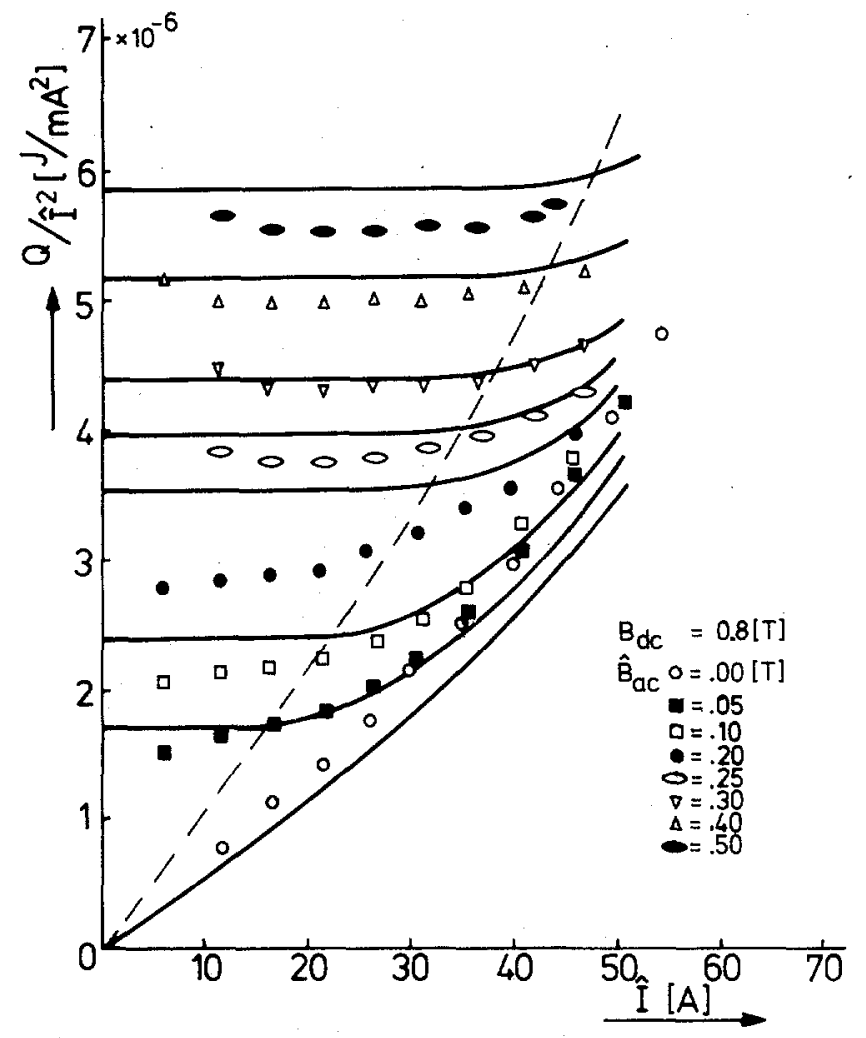

Figure 6. Measured and calculated a.c. transport current losses versus the a.c. transport current amplitude for various applied a.c. field amplitudes with a background field of $0.8 \mathrm{~T}$.

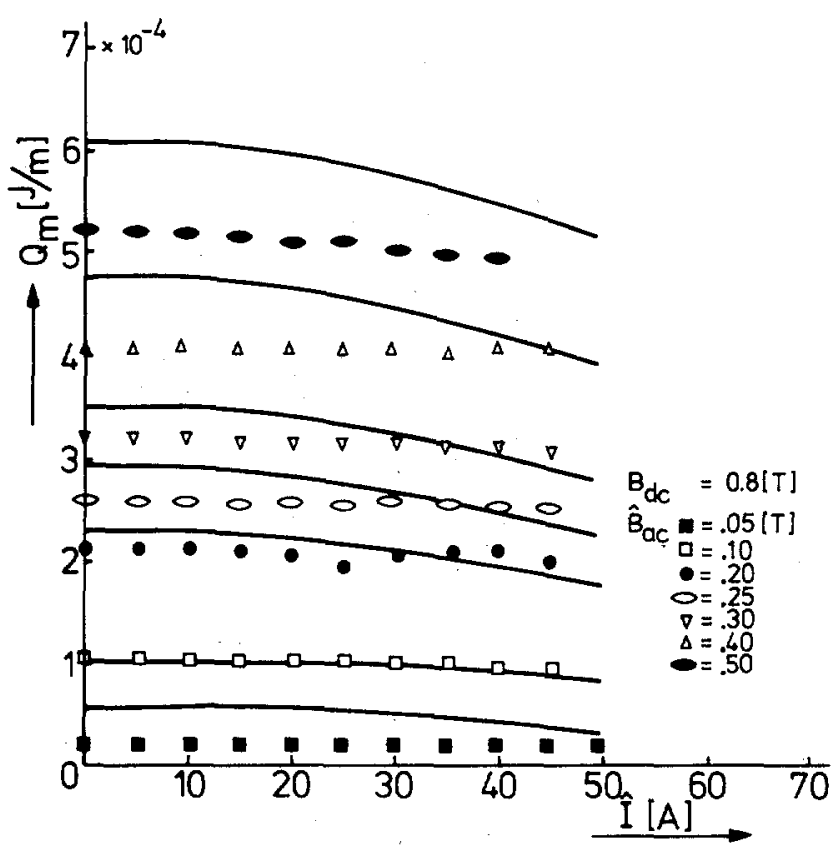

Figure 7. Measured and calculated magnetization losses versus the a.c. transport current amplitude for various applied a.c. field amplitudes with a background field of $0.8 \mathrm{~T}$.
A theoretical model provides the a.c. transport current losses for jc is constant, requiring only two parameters.

\section{Acknowledgements}

The authors wish to thank Mr. P. O'Connell for his assistance during the measuring sessions.

\section{References}

1. T. Ogasawara, M. Itoh, Y. Kubota, Y. Takahashi, $\mathrm{K}$. Yasohama and $\mathrm{K}$. Yasukochi, "Transient Field Losses in Multifilamentary Composite Conductors Carrying Transport Current", IEEE Trans. Magn., vol. MAG-17, No. 1 pp. 967, 1981.

2. T. Kawashima, F. Sumiyoshi, F. Ixie and K. Yamafuji, "Losses in a Multifilamentary superconducting wire Caused by a Simultaneous Sweep of Current and Magnetic Field", Cryogenics, pp. 313, June 1984.

3. P. Rem, J.L. de Reuver, D. Difkstra, F.P.H. van Beckum and L.J.M. van der Klundert, "The External Field Effect", Memorandum No. 478, Twente University 1984 .

4. H. London, "Alternating Current Losses in Superconductors of the Second Kind", Phys. Lett. 6, pp. 162, 1963.

5. J.L. de Reuver, P.F.C. Colenbrander, D. Dijkstra and L.J.M. van der Klundert, "Self-Field Losses in Superconducting Cixcular wires", ICEC 9, pp. 719, 1982.

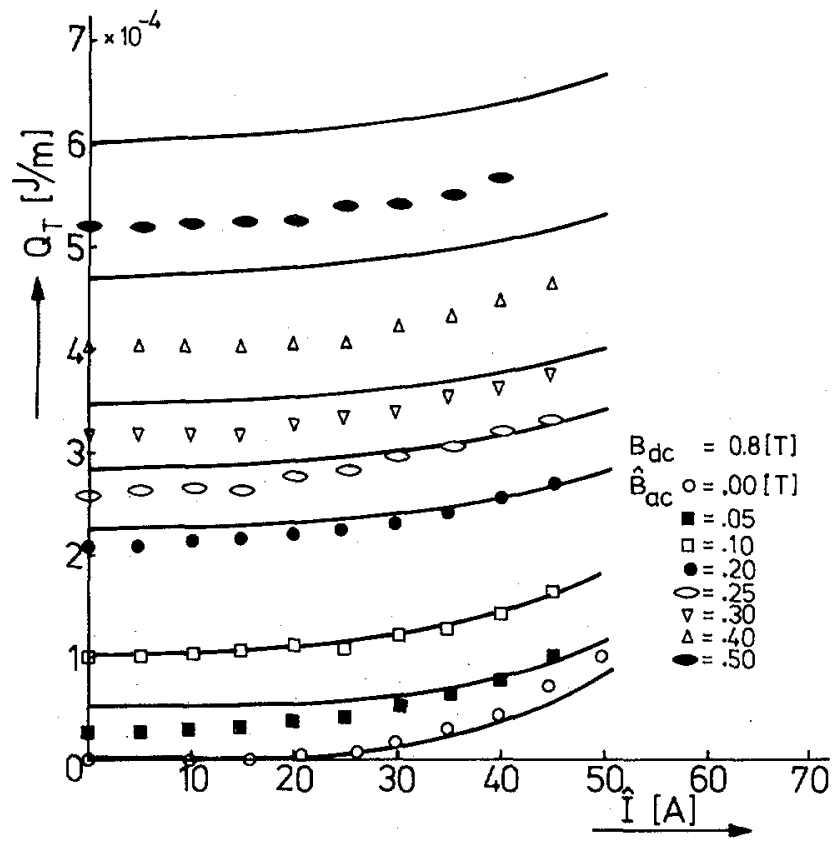

Figure 8. Measured and calculated combined losses of both a.c. transport current and a.c. field versus the a.c. transport current amplitude for various applied a.c. field amplitudes with a background field of $0.8 \mathrm{~T}$. 